\title{
Research on Database Design of Military Equipment Data Engineering
}

\author{
ZHANG Xinhui $^{[1]}, \mathrm{CHEN} \mathrm{Wei}^{[1][a]}$, and Bao Yiping ${ }^{[2]}$ \\ ( 1.Department of Aviation Ammunition, Air Force Logistics College, Xuzhou, 221000,China) \\ ( 2.Nanjing Training Battalion, Nanjing, 210000,China) \\ a.chenweijiangyiwa@126.com
}

Keywords: Military Equipment; Data Engineering; Database

\begin{abstract}
The database of military equipment data engineering is the important basis of making scientific decision for military equipment assessment, and it has the significance for developing the military equipment. The paper designed the database structure of military equipment data engineering, including the data architecture, database architecture and relational database organization, and three types were described in detail, could effectively improve the management and work efficiency of military equipment data engineering.
\end{abstract}

\section{Introduction}

The database of military equipment data engineering is to support the system demonstration and assessment of military equipment ,and it's the basic conditions for the work of the task of building major research ${ }^{[1-3]}$, which provides high reliability, a broad range of service-related military equipment database resources, greatly enhance comprehensive efficiency and the level of informatization of military equipment system assessment ${ }^{[4-5]}$. The paper designed the database structure of military equipment data engineering, could manage and exploit military equipment data, and ensured that content is complete, accurate and reliable, timely updates, effective sharing and value-added efficiency, providing effective support for the military equipment.

\section{Data Structure}

To meet the requirements of military equipment support towards related data, in compliance with the relevant standards of data engineering, infrastructure solutions, through support data field of military equipment carding and analysis of data necessary to protect classified and regular ${ }^{[6-7]}$, from the perspective of the construction of the weapons and equipment demonstration project evaluation data required construction data into fundamental data, special data and shared data altogether three categories, as shown in Fig.1.

Fundamental data: refers to shared information of military equipment data engineering, such information will not be bound by any particular data center, is the information to be used in data centers, mainly by the underlying code, the underlying data dictionary information and other equipment constitution.

Special data: refers to data centers dedicated to the support of military equipment needed to service the professional support tasks aviation data, including arms equipment data, electronic equipment data and reconnaissance equipment data, for the data center independently, only in this data Internal center sharing;

Shared data: refers to military equipment need to work together to use data, including data of military rule, military scenario data, foreign army and other relevant data, the data center, data centers shared use. 


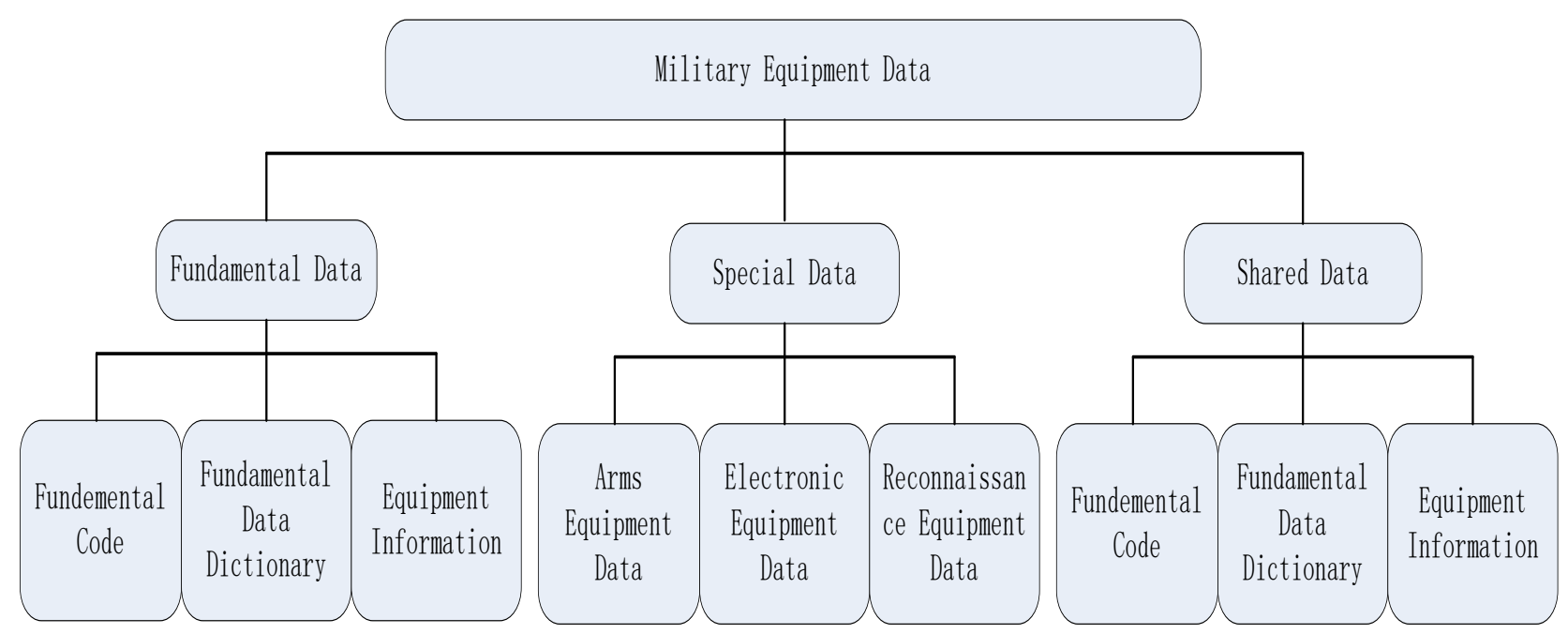

Fig.1 The partition structure of military equipment

\section{Database Structure}

Database can be divided into aerial data engineering database logic underlying database service on aviation ammunition, several common database and a number of private databases to provide a unified service standard code for the aviation ordnance engineering data ${ }^{[8-9]}$, data catalogs and data structure, data browsing, query and downloading, and various data management activities, as shown in Figure 2 constitute the database.

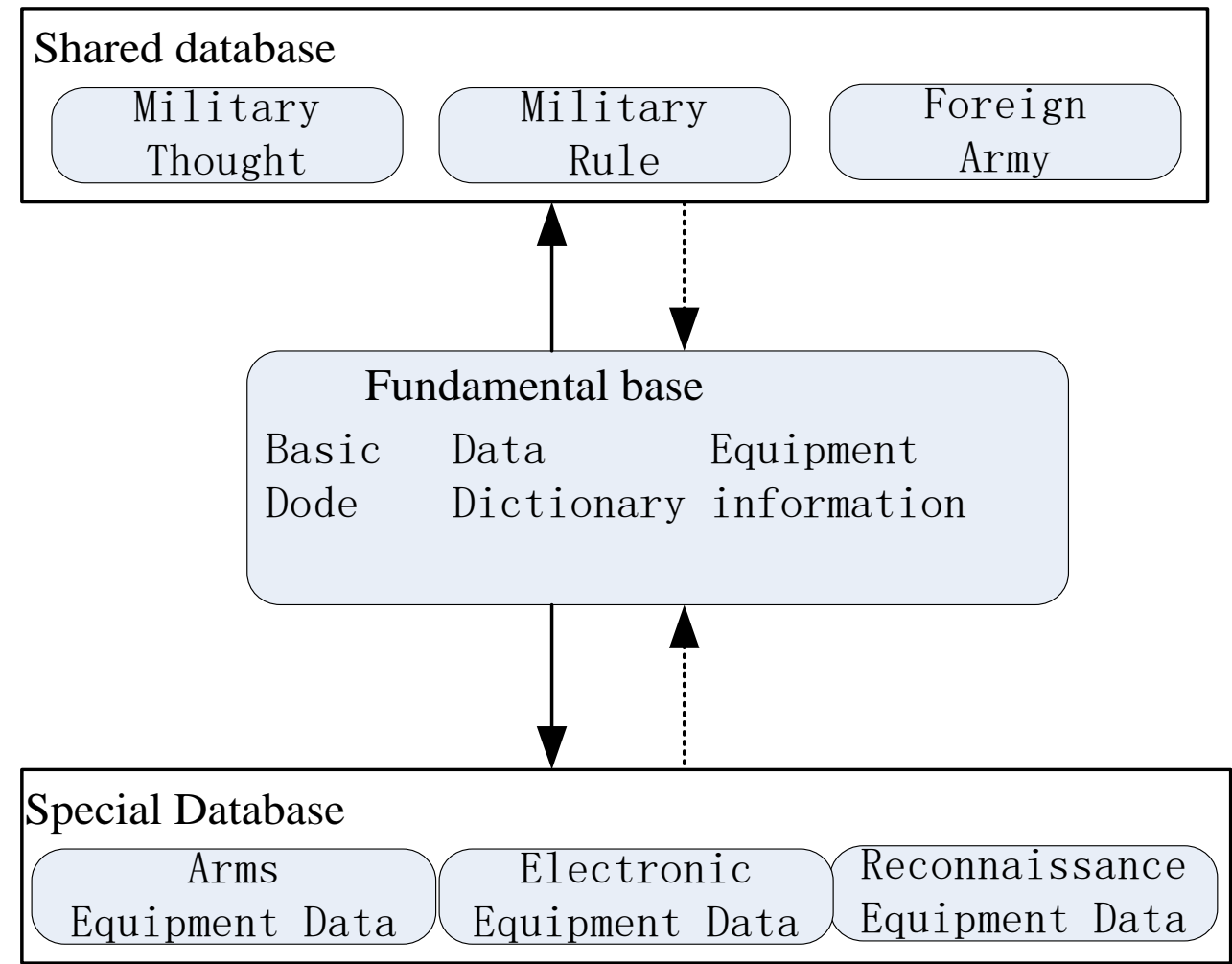

Fig. 2 The database structure of military equipment data engineering

Fundamental database: including organization, support equipment, defense research and production units, such as the underlying code, personnel attributes, unit property, equipment, property and other basic data dictionary, personnel, units, equipment and other basic information, provide basic data support for dedicated database and a common database .

Shared database: military equipment shared support work required the collection of data, browse for the data center, query and download the Assessment of the common data provide support. 
Special database: a collection of military equipment professional data support work required for internal data center to browse, search and download specific data to provide support.

The paper designed the structure of military equipment data engineering, the entire system consists of the data architecture, database architecture and relational database organization. Among them, the data architecture composed mainly of information sharing module, communication module, task queue module. System works as follows: to obtain information of military equipment data engineering, then submit this information to the blackboard Agent, Agent blackboard submit these information to the information sharing module, the monitoring Agent has a condition table, condition monitoring Agent automatically with the activation of each Agent to match the information sharing module, if the match is successful, the monitor Agent activate the Agent to obtain the required information data from the information sharing module, and submit a job node to the military equipment data in the task queue module from its knowledge base, the task queue module is responsible for setting a priority of each task node, and in descending order of priority tasks queued node according to the scheduling Agent constantly invokes the appropriate knowledge source Agent for execution processing according to the order of priority tasks while monitoring Agent blackboard constantly updated information stored until threat assessment determined situation, and finally submit the results to the human-computer interaction Agent passed to workman, support their tactical decisions.

\section{Database organization relationship}

Relying on the databases and the data centers building support data services. Which relies on the underlying database to concentrate on building a data center, and synchronized to other data centers, shared and dedicated as each database support and supplement; each shared and private databases rely on data centers, where each common database can be used as other common and supplement or "mirror" dedicated database, according to needs, providing data support; each dedicated database only to provide data services by relying on internal data center; each database in an internal data center, are realized through the analysis of different sub-libraries, refining and other forms of association; between data centers to collect reporting, issued synchronization, data mirroring, etc. to achieve the association between different databases.

Relational database organization technology has many similarities while being able to form a complementary, which provides favorable conditions for a combination of both.

Relational database organization is an efficient knowledge storage and processing tool, which is centered on the database, using the model of internal control mechanism using multiple knowledge sources to solve relevant problems. Each knowledge source are relatively independent, equivalent to an expert in a particular field, each source has its own knowledge of different forms of knowledge and algorithms, there is no uniform formal reasoning. Database organization consists of three parts: blackboard, control mechanism and sources of knowledge.

Database organization is a computer program in the computer operating system or the network environment, it can sense the external environment resources and internal state, the use of information processing module for access to information to solve reasoning judgment, to achieve their functional goals to plan their next step ${ }^{[4]}$. Agent is a certain intelligence and can actively interact with the physical surroundings, its structure is dominated by the perception module, information processing module, messaging module, intent module and action planning module.

The relationship between the various databases as follows:

a) The relationship between internal data centers of the database: the underlying database dedicated or shared database provides basic data support, dedicated or shared database of common content after refining, can form the basis of the audit data; between the internal data center based on the data of the sub-library the relationship between the associate; in addition, dedicated and shared database of relevant data resource information can be extracted form metadata stored in the metadata database. 
b) Exchange of basic data collected separately in accordance with each data center, and then to the underlying database summary report of the military principles of data centers, data centers in the army to form a complete summary of the basic data After, step by step according to the usage rights of each underlying database synchronized to the data center in order to ensure consistency between the data center infrastructure data; common database data needed to provide services to other data centers through the data center, as supplement or "mirror" other databases, but the data is not actually stored in the other databases; in addition, the contents of each data center Metabase remitted to the army of the data center, data center and then by the army of the audit, released after metadata synchronized to the associated metadata database for each data center.

\section{Summary}

Military equipment data engineering database is an important basis for military equipment support program argument. This paper designed the database structure of military equipment data engineering, including data structure, database structure and database organizational relationships, can effectively improve the management and efficiency of the military equipment data engineering service data, there is good value in practice.

\section{Acknowledgements}

This work was financially supported by the Military Equipment Data Engineering Foundation.

\section{References}

[1]Yang Boran, Zhang Shuai. Research on military training data collection[J].Naval academic2011,5:15-17.

[2]Chen Wei.Method of Event Detection Based on Dynamic Hybrid Fuzzy Logic System[C].The 2010 International Conference on Intelligent Communication Technology and Automation, 2010,

7: 661 663.

[3] M R Endsley. Theoretical Underpinnings of Situation Awareness: A Critical Review. In Situation Awareness Analysis and Measurement[J]. Lawrence Erlbaum Associates， 2000， 3 32

[4] Yansheng Lu, Pei Yue, Binbin Qu. A Simulated Evaluating System for Multi-sensor Data Fusion Algorithms [C]. IEEE Proceedings of the 5th world Congress on Intelligent Control and Automation, 2004: 3240 3244

[5] Tadaion Ali Akbar, Derakhtian Mostafa, Saeed Gazoret al.A fast multiple-source detection and localization array signal processing algorithm using the spatial filtering and ML approach[J].IEEE Transactions on Signal Processing(S1053-587x), 2007, 55(5)I: 1815 1827

[6] M. Robert, L. Craig, S. Jeffrey. Software Framework in Support of Dynamic Situation Assessment and Predictive Capabilities for JSB-RD. Enabling Technologies for Simulation Science VIII, Proceedings of SPIE, 2004, 5423: 468 478

[7] S. Greenhill. Multiple Hypotheses Situation Assessment [C]. Proceedings of the Sixth International Conference of Information Fusion, 2003, 2: 972 978

[8] Melissa Dan. Models for Threat Assessment in Networks. [D]. Dissertation of California State University, 2006, 9

[9] N. Okello, G. Thorns. Threat Assessment Using Bayesian Networks[C]. Proceedings of the Sixth International Conference of Information Fusion， 2003， 2: 1102 1109 